\title{
Computationally Efficient NMPC for Batch and Semi-Batch Processes Using Parsimonious Input Parameterization
}

\author{
Erdal Aydin $^{a, b *}$, Dominique Bonvin ${ }^{c}$, Kai Sundmacher ${ }^{a, d}$ \\ ${ }^{a}$ Max Planck Institute for Dynamics of Complex Technical Systems, Sandtorstraße 1, 39106 Magdeburg, \\ Germany \\ ${ }^{b}$ International Max Planck Research School (IMPRS) for Advanced Methods in Process and Systems \\ Engineering, Magdeburg, Germany \\ ${ }^{c}$ Laboratoire d'Automatique, Ecole Polytechnique Fédérale de Lausanne, CH-1015 Lausanne, \\ Switzerland \\ ${ }^{d}$ Otto-von-Guericke University Magdeburg, Universitätplatz 2, 39106 Magdeburg, Germany
}

* Corresponding author: aydin@ mpi-magdeburg.mpg.de ; Tel: +49 3916110 391, Fax: +49 3916110353

\begin{abstract}
The trend towards high-quality, low-volume chemical production has put more emphasis on batch and semi-batch processing due to its increased operational flexibility. The transient behavior of these processes makes their real-time optimization very challenging. In particular, the large prediction horizons required in shrinking-horizon NMPC increase the real-time computational effort due to expensive matrix factorizations.
\end{abstract}

The computational delay associated with advanced control methods is usually underestimated in theoretical studies. However, this delay may contribute to suboptimal or, worse, infeasible operation in real-life applications. This study proposes to combine a tailored parsimonious input parameterization with shrinking-horizon NMPC to reduce the real-time computational effort. 
Models of the optimal solution are used to suggest parsimonious parameterizations (especially for sensitivity-seeking arcs) that lead to computationally efficient optimization. The proposed approach is illustrated in simulation on two case studies in the presence of uncertainty, namely a batch binary distillation column and a semi-batch reactor for the hydroformylation of 1-dodecene. The results show that the tailored parsimonious shrinking-horizon NMPC (i) performs very similarly to the standard shrinking-horizon NMPC in terms of cost, (ii) is computationally much more efficient than the standard shrinking-horizon NMPC especially at the beginning of the batch, (iii) is robust to plant-model mismatch.

Keywords: Computationally efficient NMPC, shrinking-horizon NMPC, parsimonious input parameterization, batch process 


\section{Introduction}

Model predictive control (MPC) is an important tool for implementing closed-loop operation of chemical processes. It has been used extensively in industrial control applications [1]. MPC uses an explicit model (often linear) to forecast the future state behavior and compute the control actions via dynamic optimization, while taking into account process constraints. Only the first part of the computed inputs is typically applied to the plant, then the horizon is shifted by one sampling instant and the optimization repeated for the new horizon using estimates of the current states. The objective of MPC is typically the tracking of a desired trajectory. However, in recent years, the objective has been extended to the minimization of an economic cost function, thus giving rise to so-called economic MPC (eMPC) [9]. The MPC's popularity stems from the fact that it can (i) combine feedforward and feedback actions, (ii) handle process constraints, and (iii) use different performance criteria [2-10].

Batch processing has often been used to scale-up processes from the laboratory to large-scale industrial facilities [11]. ${ }^{1}$ However, in recent years, the trend in the process industry toward hightech, low-volume and high-added-value products boosted the interest in batch processing, since batch processes often represent flexible production environments. Batch processes are widely applied in the pharmaceutical, food, fine chemical and microelectronic industries. With increasing competition, the optimal operation of batch processes has moved from scheduling (for improved flexibility) to optimization (for improved profitability) [12-15].

The optimal operation of batch processes requires overcoming many challenges. Unlike continuous operation, batch processes exhibit an inherently transient behavior as well as strong nonlinearities because the process does not operate around a steady operating point.

\footnotetext{
${ }^{1}$ The wording "batch process" will be used in this work to mean both batch and semi-batch processes.
} 
Moreover, the presence of both path and terminal constraints give rise to challenging optimization problems. In addition, the lack of accurate models due to the limited amount of experimental data results in considerable uncertainty, which hinders the usage of offlinecomputed optimal profiles. In order to resolve these issues, it is vital to design effective measurement-based optimizing control laws [16-25].

Batch processes typically have constraints on end-product quality, and the ability to influence the process usually decreases with time. Often, if there is a deviation in product quality, the charge has to be discarded. Hence, shrinking-horizon nonlinear model predictive control (shNMPC) has been proposed as a successful platform for the optimal operation of batch processes, with the prediction horizon always running to the final batch time [13, 14, 26, 27]. Seki et al. [28] suggested an NMPC scheme for industrial polymerization reactors. Valappil and Georgakis [29] studied min-max and successive linearization structures for the NMPC of end-user properties in batch reactors. Nagy and Braatz [18] proposed a robust shrinking-horizon NMPC for batch crystallization, whereby parametric uncertainties are taken into account explicitly. Nagy et al. [12] studied the real-time implementation of sh-NMPC to industrial batch reactors. Mesbah et al. [30] compared different optimization algorithms for sh-NMPC of a semi-batch crystallizer. Lucia et al. [31] suggested a multi-stage NMPC for a semi-batch polymerization reactor, whereby the uncertainties are dealt with a novel scenario-tree approach. Later, Jang et al. [32] extended the multi-stage NMPC scheme by including back-off terms on path constraints. Zubov et al. [33] discussed the online implementation of NMPC to a semi-batch pilot-plant copolymerization reactor.

Unlike its linear counterpart MPC, NMPC relies on a nonlinear (usually first-principles) model to perform dynamic optimization, which results in challenging, nonconvex and constrained 
nonlinear optimization problems (NLP). Moreover, to solve these problems, sh-NMPC requires expensive matrix factorizations due to large prediction and control horizons [34, 35]. Unfortunately, there is always a certain computational delay associated with the solution to the corresponding nonconvex optimal control problems in real-time, which in turn may lead to nonnegligible feedback delay in closed-loop operation. This delay may result in suboptimal or infeasible operation [36]. Hence, it is of great importance, and still an open research topic in both academia and industry, to reduce the CPU time needed for the efficient real-time implementation of NMPCs [37]. The reader is referred to [37] for a comprehensive review of the broad class of computationally efficient NMPC methods (suboptimal, explicit, hierarchical and sensitivitybased).

Several sensitivity-based methods have been proposed in the literature to reduce the computational requirements of NLPs in the context of NMPC. These methods rely on previously computed solutions and NLP sensitivities. Diehl et al. [22] suggested a real-time iterative scheme, in which, at each sampling time, instead of a full NLP only a quadratic problem around the solution to a previous QP is solved. Another sensitivity-based method of choice is the advanced-step NMPC proposed by Zavala and Biegler [38]. In this method, the NLP problem is solved in advance with respect to a predicted initial state. Then, as soon as the new state measurements (or estimates) are available, the NLP solution is updated using a fast sensitivityupdate step and the IPOPT solver [39-41]. Successful implementations have been documented in the literature, in particular for large-scale processes $[42,43]$. However, the performance of these sensitivity-based methods is still an open question for batch processes that are characterized by highly nonlinear effects and large perturbations. For a detailed review of the recent advances in the sensitivity-based NMPC, the reader is referred to [44]. 
The optimal inputs in batch processes are characterized by the presence of various arcs. An optimal arc is either determined by an active input bound $\left(u_{\min }, u_{\max }\right)$ or a path constraint $\left(u_{\text {path }}\right)$, or it is inside the feasible region as a sensitivity-seeking arc $\left(u_{\text {sens }}\right)$ [14]. It is usually difficult to accurately compute the fine shapes of sensitivity-seeking arcs due to their lack of sensitivity. As a result, simple models of the optimal solution can be introduced, in which the inputs, and most importantly the sensitivity-seeking arcs $u_{\text {sens }}$, are parameterized parsimoniously using switching times and low-order polynomials. This way, the number of decision variables and the complexity of the optimization problem are reduced significantly. In other words, instead of a full NLP, a parsimoniously parameterized NLP is solved, which reduces the computational load of the corresponding nonconvex dynamic optimization problem. Hence, the required CPU time is expected to decrease significantly, without affecting much the optimal cost [45-48].

The main contribution of this work is to detail the application of these parsimonious input parameterizations in the context of sh-NMPC. As stated earlier, the optimization is performed at each sampling instant for the full time horizon, but only the first part of the optimal inputs is applied to the process. Although the resulting "parsimonious sh-NMPC" only approximates the fine shapes of the optimal inputs at each sampling instant, the optimal closed-loop behavior might be captured accurately. In addition, since the full time horizon is taken into account, the loss in ability to influence the batch outcome can be prevented, while still having a significant reduction in CPU time.

The paper is structured as follows. Section 2 illustrates the proposed methodology and its combination with sh-NMPC. Section 3 presents two case studies, namely, a batch binary distillation with terminal purity constraints and a semi-batch hydroformylation reactor with path constraints. Finally, Section 4 concludes the study. 


\section{Shrinking-Horizon NMPC}

\subsection{Standard sh-NMPC}

Standard sh-NMPC of batch processes requires the solution to the corresponding optimal control problem online at each iteration:

$$
\begin{array}{cc} 
& \min _{u(t)} J=\phi\left(x\left(t_{f}, \theta\right)\right) \\
\text { s.t. } \quad \dot{x}(t) & =F(x(t), u(t), \theta), \quad x\left(t_{k}\right)=x_{k} \\
& S(x(t), u(t), \theta) \leq 0, \quad T\left(x\left(t_{f}\right), \theta\right) \leq 0, \quad t \in\left[t_{k}, t_{f}\right]
\end{array}
$$

where $t_{k}$ is the time at the $k$-th iteration, $F$ is the system of equations, $J$ is the scalar performance index expressed as a function of the states at the final time $t_{f}, \phi$ is the objective function, $x(t)$ is the state vector with the initial conditions $x_{k}$ at $t_{k}, u(t)$ is the input vector, $S$ is the vector of inequality path constraints that include input bounds, $T$ is the vector of inequality terminal constraints, and $\theta$ is the vector of uncertain parameters associated with plant-model mismatch. After solving Problem (1), the first part $u\left[t_{k}, t_{k}+\delta\right]$ of the optimal inputs is applied to the plant, the horizon is shrunk by the sampling interval $\delta$, the new states $x_{k+1}$ are estimated, and a new optimal control problem is solved. These steps are repeated until the final batch time is reached.

Direct and indirect methods exist in the literature to solve Problem (1) $[15,26,49,50]$. The input profiles are typically discretized as $u(t)=\mathrm{U}(\mathrm{U})$, where $\mathrm{U} \in \Re^{\left(n_{u} \times N\right)}$ is an input matrix that contains $N$ discrete values for the $n_{u}$ inputs. In an earlier publication, it was observed that at least $N=100$ is required to obtain reliable optimal profiles for the batch and semi-batch problems investigated in this work [26].

Accordingly, the standard sh-NMPC algorithm can be formulated as follows: 


\section{Standard sh-NMPC Algorithm}

Consider the optimization Problem (1). Set $k=0$ and specify $x_{0}$. Specify $t_{f}$ and $\delta$.

while $t_{k} \in\left[0, t_{f}\right] \mathbf{d o}$

1. Measure/estimate $x_{k}$ and assign $x\left(t_{k}\right):=x_{k}$

2. Solve Problem (1) for the input matrix $\mathrm{U}$, which gives $u\left[t_{k}, t_{f}\right]=\mathrm{U}(\mathrm{U})$

3. Apply $u\left[t_{k}, t_{k}+\delta\right]$ to the plant and wait for $t_{k+1}=t_{k}+\delta$

4. Set $k:=k+1$

end do

Numerical optimization schemes often require fine input discretization levels to be able to compute accurate solutions. In addition, since the complexity of the optimization problem increases exponentially with the time horizon due to the matrix factorizations required in the solution steps $[35,51]$, the CPU time required to solve the sh-NMPC problem may turn out to be large, especially at the beginning of the batch when the time horizon is the largest. On the other hand, the expensive matrix factorizations can be avoided by decreasing the number of decision variables using a parsimonious input parameterization, while keeping the full length of the time horizon.

\subsection{Parsimonious sh-NMPC}

The strategy behind building the parsimonious input parameterization starts with the offline solution to Problem (1). It is typically assumed that the uncertainty does not change the types and sequence of optimal arcs in closed-loop operation, which is reasonable for batch processes [16, 52]. Given the optimal solution structure and the input bounds $\left(u_{\min }, u_{\max }\right)$, the sensitivityseeking arcs $\left(u_{\text {sens }}\right)$ can be approximated using polynomial profiles and adjustable switching times between $\operatorname{arcs}[21,45,46,53]$. 
Given the optimal solution structure, it is possible to reformulate the optimization problem using a parsimonious input parameterization of the form $u(t)=\mathrm{U}(\pi)$. For example, a sensitivityseeking arc can be expressed as a linear arc between the two switching times $t_{1}$ and $t_{2}$. This results in $\pi=\left(t_{1}, t_{2}, a_{1}, a_{2}\right)^{T}$, with the parsimonious input model:

$$
\mathcal{U}(\pi)= \begin{cases}u_{\max } & \text { if } 0 \leq t<t_{1} \\ a_{1}+\frac{a_{2}-a_{1}}{t_{2}-t_{1}}\left(t-t_{1}\right) & \text { if } t_{1} \leq t<t_{2} \\ u_{\min } & \text { if } t_{2} \leq t<t_{f}\end{cases}
$$

The reformulated optimal control problem to be solved at each sampling instant reads:

$$
\begin{array}{cc} 
& \min _{\pi} \tilde{J}=\phi\left(x\left(t_{f}, \theta\right)\right) \\
\text { s.t. } \quad \dot{x}(t) & =\tilde{F}(x(t), \mathcal{U}(\pi), \theta), \quad x\left(t_{k}\right)=x_{k} \\
& \tilde{S}(x(t), \mathcal{U}(\pi), \theta) \leq 0, \quad T\left(x\left(t_{f}\right), \theta\right) \leq 0, \quad t \in\left[t_{k}, t_{f}\right]
\end{array}
$$

where $\pi$ is the new vector of decision variables, $\mathcal{U}(\pi)$ is the input vector given by Eq. (2), $\tilde{F}$ represents the system of equations expressed in terms of $\mathcal{U}(\pi)$ instead of $u(t), \tilde{J}$ is the scalar performance index for Problem (3), $\tilde{S}$ is the vector of inequality path constraints expressed in terms of $U(\pi)$ instead of $u(t)$. 
Accordingly, the parsimonious sh-NMPC algorithm can be formulated as follows:

\section{Parsimonious sh-NMPC Algorithm}

I. Solve Problem (1) numerically offline for the nominal parameter values $\theta_{0}$.

II. Build the parsimonious input model $\mathcal{U}(\pi)$ by parameterizing the inputs with respect to the switching times and low-order polynomials.

III. Set $k=0$ and specify $x_{0}$. Specify $t_{f}$ and $\delta$.

while $t_{k} \in\left[0, t_{f}\right] \mathbf{d o}$

1. Measure/estimate $x_{k}$ and assign $x\left(t_{k}\right):=x_{k}$

2. Solve Problem (3) for the decision variables $\pi$, which gives $u\left[t_{k}, t_{f}\right]=\mathcal{U}(\pi)$

3. Apply $u\left[t_{k}, t_{k}+\delta\right]$ to the plant and wait for $t_{k+1}=t_{k}+\delta$

4. set $\mathrm{k}:=\mathrm{k}+1$

end do

Remark 1. The polynomials used in the parsimonious input parameterization are very problem specific, with piecewise-constant or piecewise-linear functions often resulting in accurate approximations to the sensitivity-seeking arcs. Problem-specific information regarding the parsimonious parameterization will be detailed in the two case studies of next section.

Remark 2. Depending on their relative degree, the inputs that activate the path constraints $u_{\text {path }}$ can sometimes be computed online using the model equations, that is, without any optimization $[15,48]$. Another alternative is to track the corresponding path constraints with the help of feedback controllers using $u_{p a t h}(t)$ as manipulated variables [52]. 


\section{Case Studies}

To illustrate the application of parsimonious sh-NMPC to batch processes, two case studies are selected. The first example is a batch binary distillation column with terminal purity constraints, while the second one is a semi-batch hydroformylation reactor with path constraints. The nominal models and parameters of both examples are taken from [26]. In order to test the performance and robustness of the controllers, closed-loop simulations are performed under parametric uncertainties. The standard sh-NMPC cases are solved using a direct simultaneous method. The direct simultaneous method uses orthogonal collocation on uniform finite elements for both state and input discretization. The degree of the interpolating polynomials is 4 . The number of finite elements is problem specific and will be given in the next sections. The CasADi toolbox and Matlab Simulink are used for both sh-NMPC methods [41, 54].

\subsection{Batch Binary Distillation with Terminal Purity Constraints}

Consider a batch distillation column with three equilibrium plates, in which components A and B (more volatile) are separated from each other. The operational goal is the maximization of the amount $\mathrm{B}$ in the distillate, while satisfying two terminal constraints, namely, the top composition of B must be at least $80 \mathrm{~mol} \%$ and the bottom composition of B must be at most 20 mol \%. The only path constraint is on the input variable, namely, the reflux ratio. The schematic of the column can be found in [26]. The optimal control problem to be solved online in the context of sh-NMPC reads (the material balances are written in terms of the more volatile component B): 


$$
\max _{r(t), t_{f}} J=D\left(t_{f}\right)
$$

s.t.

$$
\begin{gathered}
\dot{D}=V(1-r) ; \quad D\left(t_{k}\right)=D_{k} \\
\dot{B}=V(r-1) ; \quad B\left(t_{k}\right)=B_{k} \\
\dot{n}_{B}=\dot{x}_{B} B+\dot{B} x_{B}=V\left(-y_{B}+r x_{1}\right) ; \quad n_{B}\left(t_{k}\right)=n_{B k} \\
\dot{n}_{1}=\dot{x}_{1} M=V\left(y_{B}-y_{1}+r\left(x_{2}-x_{1}\right)\right) ; \quad n_{1}\left(t_{k}\right)=n_{1_{k}} \\
\dot{n}_{2}=\dot{x}_{2} M=V\left(y_{1}-y_{2}+r\left(x_{3}-x_{2}\right)\right) ; \quad n_{2}\left(t_{k}\right)=n_{2_{k}} \\
\dot{n}_{3}=\dot{x}_{3} M=V\left(y_{2}-y_{3}+r\left(y_{3}-x_{3}\right)\right) ; \quad n_{3}\left(t_{k}\right)=n_{3_{k}} \\
\dot{n}_{D}=V(1-r) y_{3} ; \quad n_{D}\left(t_{k}\right)=n_{D_{k}} \\
y_{m}=\frac{\alpha x_{m}}{1+(\alpha-1) x_{m}} ; \quad m=B, 1, \ldots, 3 \\
x_{D}\left(t_{f}\right)=n_{D}\left(t_{f}\right) / D\left(t_{f}\right) \geq 0.8 \\
x_{B}\left(t_{f}\right)=n_{B}\left(t_{f}\right) / B\left(t_{f}\right) \leq 0.2 \\
3 \mathrm{~h} \leq t_{f} \leq 3.25 \mathrm{~h} \\
0 \leq r(t) \leq 1 \quad, \quad t \in\left[t_{k}, t_{f}\right]
\end{gathered}
$$

where $t_{k}$ is the time at the $k$-th iteration, $B_{k}$ the charge, $n_{B_{k}}$ the moles of B in the charge, $n_{m}$ the moles of B in the liquid phase on the $m$-th tray, $y_{m}$ the mole fraction of $\mathrm{B}$ in the vapor phase leaving the $m$-th tray, $n_{D}$ the moles of B in the distillate tank, $x_{D}$ the mole fraction of $\mathrm{B}$ in the distillate tank, $n_{B}$ the moles of $\mathrm{B}$ in the bottoms, $x_{B}$ the mole fraction of $\mathrm{B}$ in the bottoms, $\alpha$ the relative volatility, $M$ the liquid hold-up on each tray, and $t_{f}$ the free final time. Because of the assumption of total condensation, the composition of the refluxed liquid is equal to the vapor composition leaving the upper plate. It is also assumed that all plates are initially charged with 
the same liquid mixture as the reboiler. The nominal model parameters and the initial conditions are given in Table 1.

Table 1. Nominal model parameters and initial conditions for the batch distillation.

\begin{tabular}{|c|c|}
\hline Vapor flowrate, $V$ & $50 \mathrm{kmol} / \mathrm{h}$ \\
\hline Relative volatility, $\alpha$ & 2.35 \\
\hline Initial charge, $B_{0}$ & $115 \mathrm{kmol}$ \\
\hline Concentration of B in the charge, $x_{B_{0}}$ & 0.4 \\
\hline Molar hold-up per plate, $M$ & $5 \mathrm{kmol}$ \\
\hline
\end{tabular}

It is assumed that state feedback information is available in order to focus only on the computational aspects of the optimization scheme. This is a reasonable assumption considering the advances in state estimation and online spectroscopy $[43,55,56]$. In addition, since the final time is not fixed but represents a degree of freedom in the optimization problem, a time transformation is introduced in the ODEs to have a unity normalized time and $t_{f}$ as a decision variable.

\subsubsection{Nominal Open-Loop Optimal Policy}

The optimal control Problem (4) is first solved offline using the parameters given in Table 4. The optimal profiles, computed using a direct simultaneous method and 100 piecewise-constant elements, are given in Fig. 1. The optimal cost is 44.69 [kmol]. 

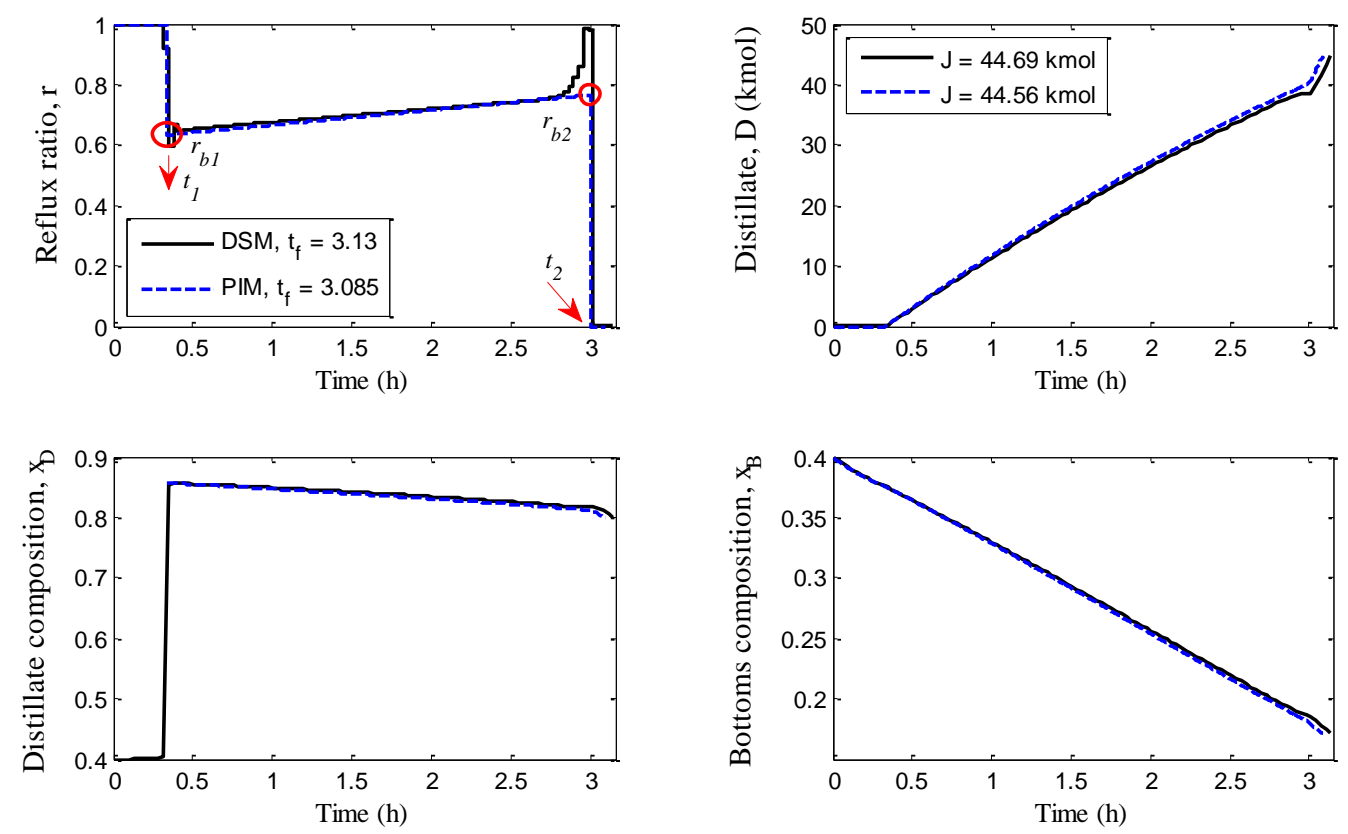

Figure 1. Optimal open-loop profiles for Problem (4) obtained with a direct simultaneous method (DSM) and a parsimonious input model (PIM).

Fig. 1 shows that the optimal solution starts with total reflux to increase the purity at the top of the column. Then, a sensitivity-seeking arc represents the best compromise between producing more distillate and satisfying the required terminal purities. Finally, a no-reflux third arc recovers the high-purity material that is still at the top of the column.

Analyzing the optimal input profile, a parsimonious input model can be proposed, in which the sensitivity-seeking arc varies linearly between the two switching times $t_{1}$ and $t_{2}$. Furthermore, the values of $r_{\text {sens }}$ at the switching times are also considered as the decision variables $r_{b 1}$ and $r_{b 2}$, because $r_{s e n s}$ does not start at 1 and end at 0 . Accordingly, the new vector of decision variables for this parsimonious input model is $\pi=\left(t_{1}, t_{2}, r_{b 1}, r_{b 2}, t_{f}\right)^{T}$, and the parameterized reflux ratio reads: 


$$
r(\pi)= \begin{cases}1 & \text { if } 0 \leq t<t_{1} \\ r_{b 1}+\frac{r_{b 2}-r_{b 1}}{t_{2}-t_{1}}\left(t-t_{1}\right) & \text { if } t_{1} \leq t<t_{2} \\ 0 & \text { if } t_{2} \leq t<t_{f}\end{cases}
$$

The optimal profiles obtained with this parsimonious input model are also shown in Fig. 1, with an optimal cost of 44.56 [kmol]. It is observed that the nominal and approximated open-loop optimal profiles are very similar.

\subsubsection{Closed-Loop Simulations with sh-NMPC}

In order to check the performances of the two sh-NMPC schemes, uncertainty is added to the relative volatility parameter $\alpha$, which is allowed to vary randomly from batch to batch between $15 \%$ and $0 \%$. In addition, the vapor flowrate $V$ is randomly perturbed by $\pm 3 \mathrm{kmol} / \mathrm{h}$ within the batch. The controller sampling time is $1 \mathrm{~min}$.

Firstly, the performances of standard and parsimonious sh-NMPC are compared for one particular batch. The optimal closed-loop behaviors are given in Fig. 2, which shows that very similar performance can be achieved with both methods. In other words, the closed-loop optimal behavior can be approximated accurately using the parsimonious input model. 

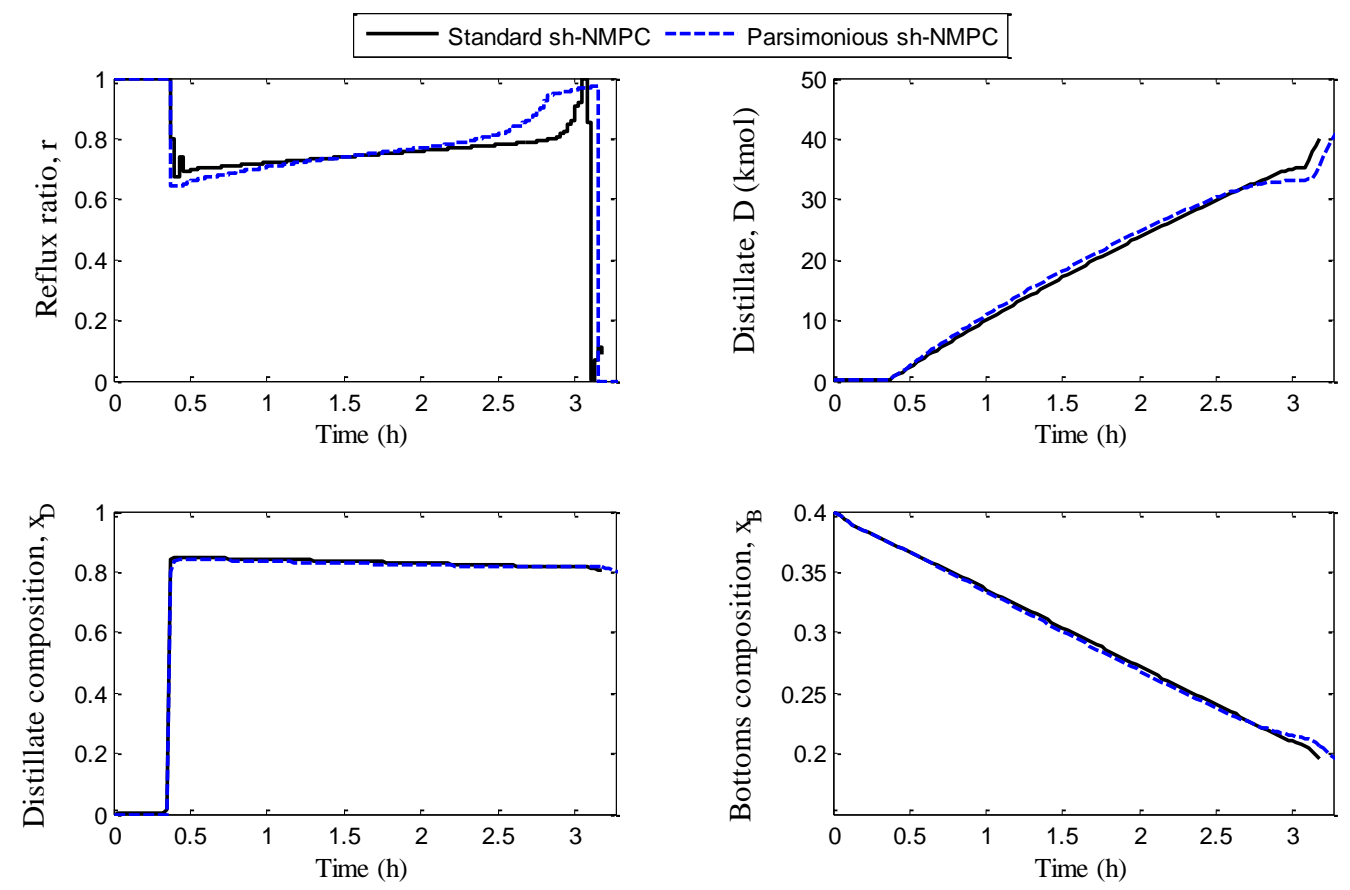

Figure 2. Optimal closed-loop profiles for Problem (4) obtained with standard and parsimonious sh-NMPC.

On the other hand, Fig. 3 indicates that parsimonious sh-NMPC requires significantly less CPU time and is much faster than standard sh-NMPC, especially at the beginning of the batch. 


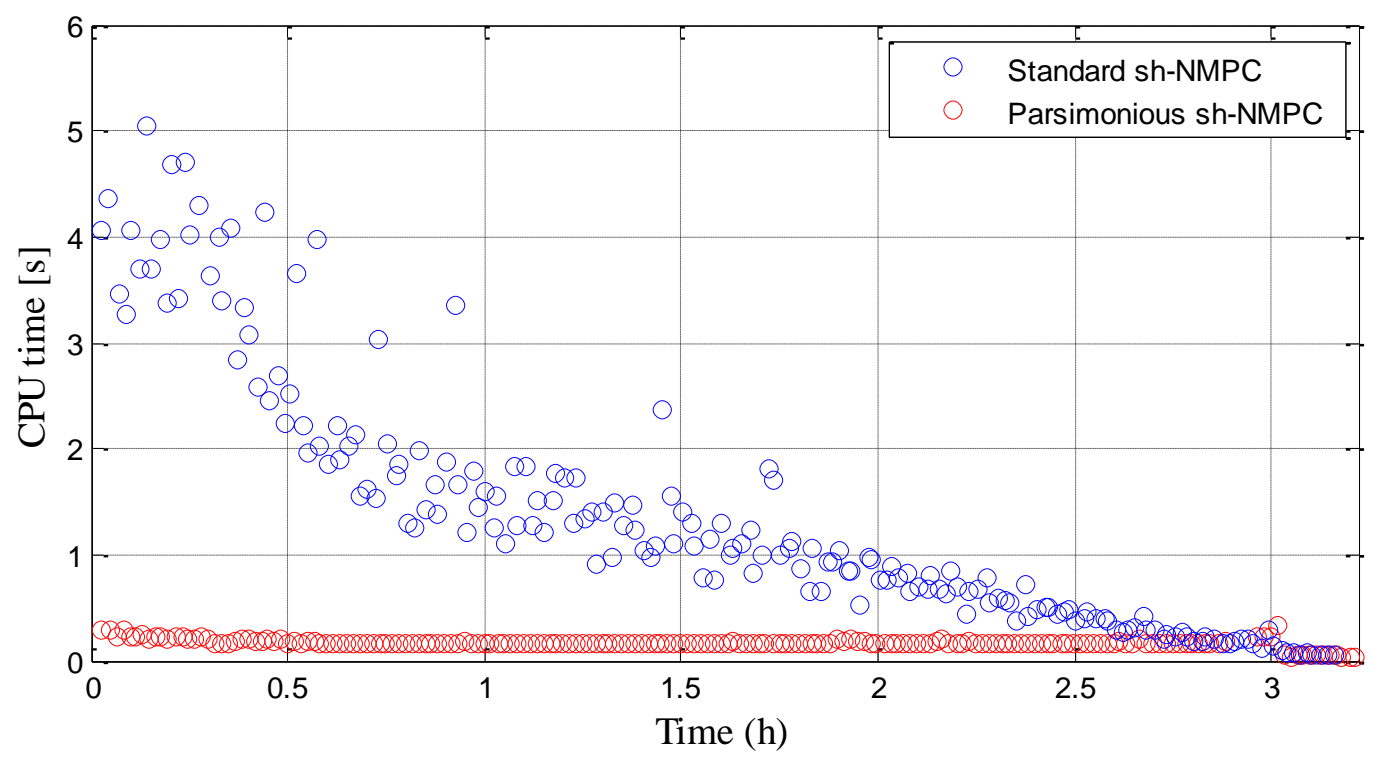

Figure 3. Computational times with standard and parsimonious sh-NMPC.

Finally, to validate the robustness and check the performance of parsimonious sh-NMPC for different uncertainty realizations, 40 closed-loop simulations were performed. In all cases, the simulation is stopped as soon as the desired purity is achieved. The results are given in Fig. 4. Note that the parsimonious sh-NMPC results in feasible operation. Furthermore, with reduced CPU time at each iteration, faster sampling and control can be used, which in turn helps deal with plant-model mismatch. This way, parsimonious sh-NMPC can outperform standard sh-NMPC in real-life implementation. 

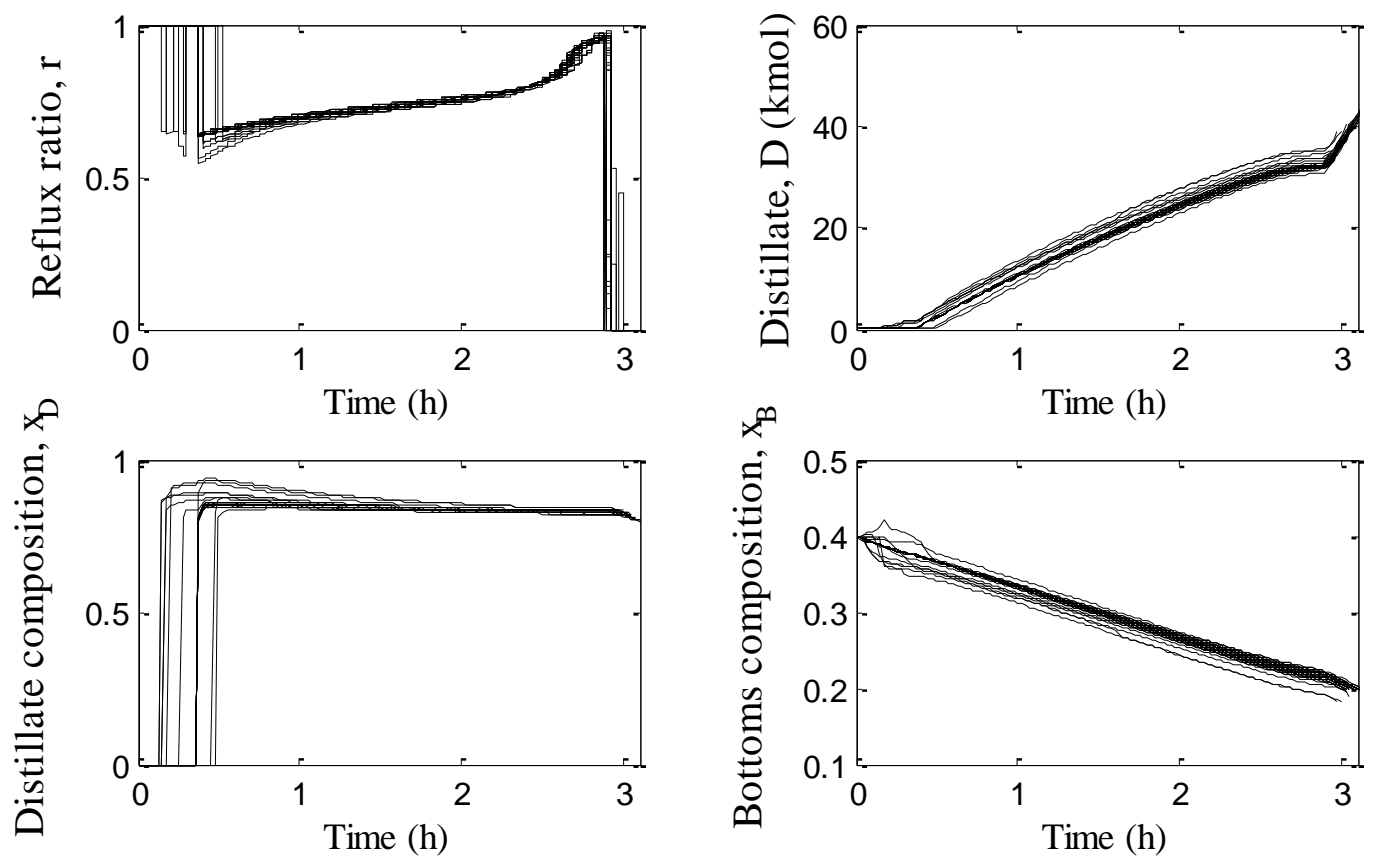

Figure 4. Optimal closed-loop profiles for Problem (4) obtained with parsimonious sh-NMPC for 40 different batches.

\subsection{Semi-Batch Reactor for the Hydroformylation of 1-Dodecene}

Long-chain olefins exhibit potential as renewable feedstocks for existing petrochemical production plants. Hydroformylation is an alternative way of processing these feedstocks into aldehydes, which are valuable intermediates. This section investigates the application of shNMPC to a two-phase fed-batch reactor for the hydroformylation of 1-dodecene (nC12en) in the presence of uncertainty.

A stirred-tank reactor with the dosing of syngas $\left(\mathrm{H}_{2}+\mathrm{CO}\right)$ is used for the operation. The manipulated variables are the reactor temperature $T(t)$ and the feedrate of syngas $u(t)$. The gas and liquid phases are modeled as ideally mixed phases, and the model parameters have been estimated and validated using experimental data [57]. The liquid volume $V_{l i q}$ and the gas volume 
inside the reactor $V_{\text {gas }}$ are assumed to be constant $(900 \mathrm{~mL}$ each). The operational objective is to maximize the amount of $\mathrm{n}$-tridecanal (nC13al) at the end of the batch. Input bounds and limits on the total pressure in the gas phase represent the path constraints that should be met throughout the operation. Earlier studies showed that plant-model mismatch affects the process conditions and feasibility significantly and, therefore, should be taken into account $[58,59]$. The species indices are as follows: 1: $\mathrm{H}_{2}, 2: \mathrm{CO}, 3: n C 12 e n, 4: i C 12 e n, 5:$ nc13al, 6: ic13al, 7: nC12an. Only two species are present in the gas phase, namely, $\mathrm{H}_{2}$ and $\mathrm{CO}$. The reaction network can be found in [57]. Note that the reaction takes place in the liquid phase.

The optimal control problem to be solved online at each sh-NMPC iteration reads $[58,59]$ :

$$
\begin{aligned}
& \max _{u(t), T(t)} J=c_{n c 13 a l}\left(t_{f}\right) \quad t \in\left[t_{k}, t_{f}\right] \\
& \text { s.t } \quad \dot{c}_{i}=j_{i}^{G L}+c_{c a t} M_{c a t} \sum_{j \in \mathrm{R}} v_{j, i} r_{j} ; \quad c_{i}\left(t_{k}\right)=\hat{c}_{i, k} ; \quad \mathrm{i}=1,2, \ldots, 7 \\
& \dot{p}_{i}=\frac{R T}{V_{\text {gas }}}\left(u x_{i}-V_{\text {liq }} j_{i}{ }^{G L}\right) \quad(i \in g a s) ; p_{i}\left(t_{k}\right)=p_{i, k} ; x_{i}=0.5\left(\frac{\mathrm{mol}}{\mathrm{mol}}\right) ; \mathrm{i}=1,2 \\
& j_{i}^{G L}=\left\{\begin{array}{lr}
\left(k_{L} a\right)_{i}\left(c_{i}^{*}-c_{i}\right), & (\text { if } i \in \text { gas }) ; \mathrm{i}=1,2 \\
0, & (\text { else }) ; \mathrm{i}=3,4, \ldots, 7
\end{array}\right. \\
& r_{1}=\frac{k_{1,0}(T) c_{n C 12 e n} c_{H_{2}} c_{C O}}{1+K_{1,1} c_{n C 12 e n}+K_{1,2} c_{n C 13 a l}+K_{1,3} c_{H_{2}}} \\
& r_{2}=\frac{k_{2,0}(T)\left(c_{n C 12 e n}-\frac{c_{i C 12 e n}}{K_{p, 2}}\right)}{1+K_{2,1} c_{n C 12 e n}+K_{2,2} c_{i C 12 e n}} \\
& r_{3}=\frac{k_{3,0}(T)\left(c_{n C 12 e n} c_{H_{2}}-\frac{c_{n C 12 a n}}{K_{p, 3}}\right)}{1+K_{3,1} c_{n C 12 e n}+K_{3,2} c_{n C 13 a n}+K_{3,3} c_{H_{2}}} \\
& r_{4}=k_{4,0}(T) c_{i C 12 e n} c_{H_{2}} \\
& r_{5}=k_{5,0}(T) c_{i C 12 e n} c_{H_{2}} c_{C O}
\end{aligned}
$$




$$
\begin{aligned}
& r_{6}=k_{6,0}(T) c_{n C 12 e n} c_{H_{2}} c_{C O} \\
& k_{j}(T)=k_{0, j} \exp \left(-\frac{E_{A, j}}{R}\left(\frac{1}{T}-\frac{1}{T_{\text {ref }}}\right)\right) \\
& K_{p, j}=\exp \left(\frac{-\Delta G_{j}}{R T}\right) \\
& -\Delta G_{j}=a_{0, j}+a_{1, j} T+a_{2, j} T^{2} \\
& c_{c a t}=\frac{\gamma c_{c a t, t o t}}{1+K_{c a t, 1} c_{C O}{ }^{K_{c a t, 3}}+K_{c a t, 2} \frac{c_{C O} K_{c a t, 3}}{c_{H_{2}}}} \\
& c_{i}^{*}=\frac{p_{i}}{H_{i}} \\
& H_{i}=H_{i}{ }^{0} \exp \left(\frac{-E_{A, H, i}}{R T}\right) \\
& p_{\text {total }}(t)=p_{H_{2}}(t)+p_{C O}(t) \\
& 1 \text { bar } \leq p_{\text {total }}(t) \leq 20 \text { bar } \\
& 368.15 \mathrm{~K} \leq T(t) \leq 388.15 \mathrm{~K} \\
& \frac{\left|T(t)-T\left(t_{k}\right)\right|}{t-t_{k}} \leq \Delta T_{\max } \\
& c_{n c 13 a l}\left(t_{f}\right) \leq 0.8 \quad t \in\left[t_{k}, t_{f}\right]
\end{aligned}
$$

where $t_{k}$ is the time at the $k$-th iteration, $i$ represents the component index $(\mathrm{i}=1,2, \ldots, 7$ for the liquid phase, and $\mathrm{i}=1,2$ for the gas phase), $j$ is the reaction index, $\mathrm{R}$ is the reaction set, $\hat{c}_{i, k}$ is the estimated concentration of component $i$ in the liquid phase, $p_{i, k}$ is the partial pressure of Gas $i$ in the gas phase, and $\gamma$ is catalyst activity. The fixed final time $t_{f}$ is $70 \mathrm{~min}$. The nominal model parameter values are given in Table 2. 
Table 2. Nominal parameter values and corresponding variations for the hydroformylation process: $\left(k_{L} a\right)_{i}$ varies within batch, while $k_{i, 0}$ and $\gamma$ vary from batch to batch.

\begin{tabular}{|c|c|c|c|}
\hline Parameter & $\begin{array}{c}\text { Nominal Value } \\
\text { [57] }\end{array}$ & $\begin{array}{l}\text { Minimal } \\
\text { Value }\end{array}$ & $\begin{array}{l}\text { Maximal } \\
\text { Value }\end{array}$ \\
\hline$\left(k_{L} a\right)_{1}$ & 9.57 & 8.57 & 10.57 \\
\hline$\left(k_{L} a\right)_{2}$ & 7.08 & 6.08 & 8.08 \\
\hline$k_{1,0}$ & 4.904 & 3.8 & 6.0 \\
\hline$k_{2,0}$ & 4.878 & 3.78 & 5.98 \\
\hline$k_{3,0}$ & 2.724 & 1.72 & 3.72 \\
\hline$k_{4,0}$ & 2.958 & 1.8 & 4.0 \\
\hline$k_{5,0}$ & 3.702 & 2.6 & 4.8 \\
\hline$k_{6,0}$ & 3.951 & 2.8 & 5.0 \\
\hline$\gamma$ & $100 \%$ & $80 \%$ & $100 \%$ \\
\hline
\end{tabular}

The optimal reactor temperature calculated by sh-NMPC serves as the setpoint for a thermostat, where a PID controller regulates the reactor temperature by adjusting the flowrate of the heating or cooling fluid. Hence, a rate constraint on the temperature change is introduced (with $\Delta T_{\max }=0.0233 \mathrm{~K} / \mathrm{s}$ ) in order for the controller to be capable of reaching the setpoint before the next sh-NMPC iteration. This rate constraint term depends on the heating/cooling capacity of 
the thermostat. On the other hand, the optimal feedrate can be applied directly to the reactor [25, $60]$.

\subsubsection{Nominal Open-Loop Optimal Policy}

Problem (6) is solved offline for the nominal parameter values. The input parameterization uses 500 piecewise-constant elements. The optimal profiles are shown in Fig. 5. It is seen that (i) the optimal solution exhibits a single arc for the feedrate $u$, and (ii) the upper pressure limit is always active, $p_{\text {total }}(t)=20$ bar. Furthermore, the optimal temperature profile starts at the lower limit $\left(T_{\min }\right)$ to favor the desired reactions, then follows a sensitivity-seeking arc ( $\left.T_{\text {sens }}\right)$ that expresses the compromise between producing as much as nC13al as possible without producing too much side products, and ends up at the upper limit $\left(T_{\max }\right)$ to suppress the undesired reactions. The optimal cost with the fully parameterized NLP is 0.591 [mol/L]. 

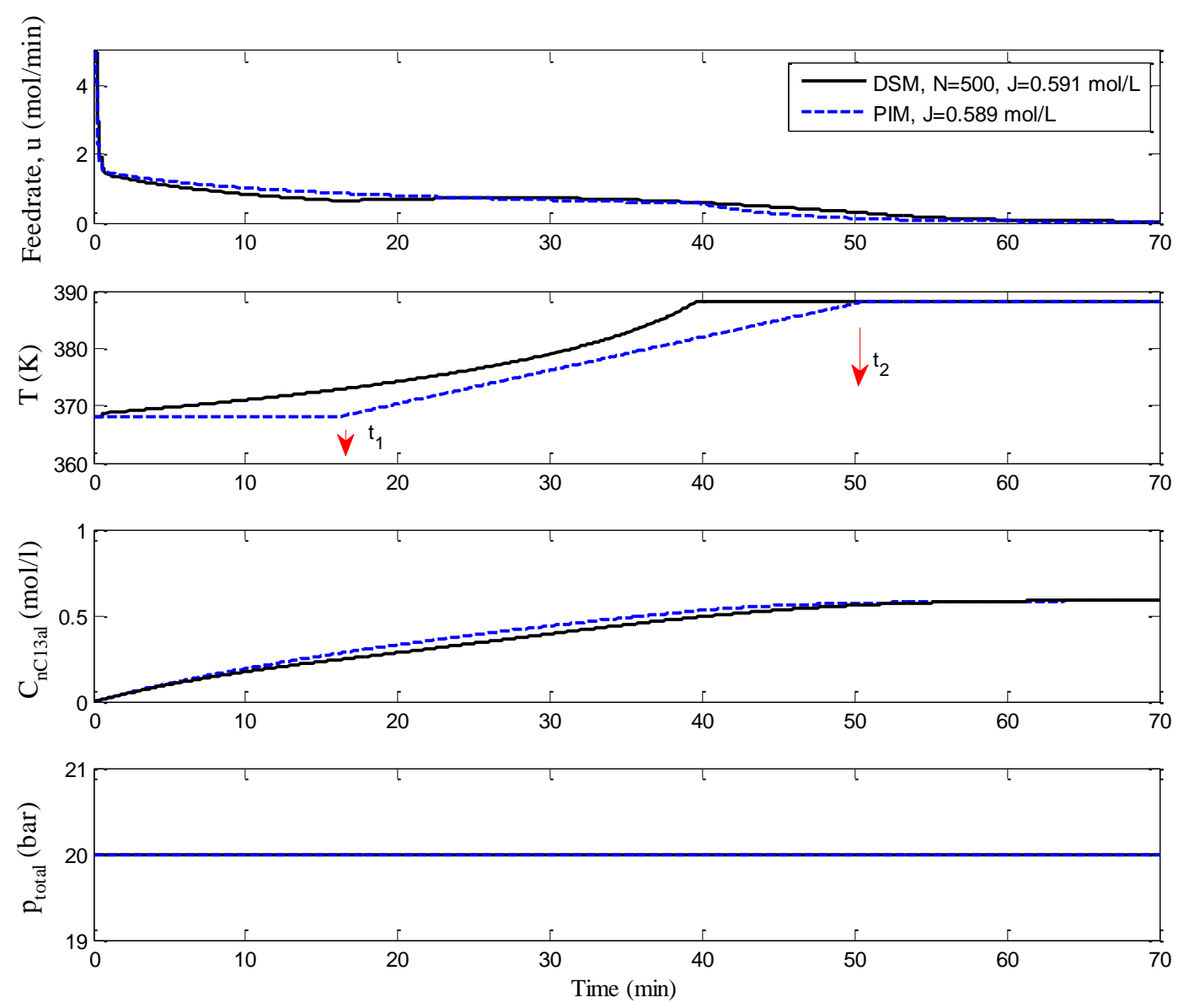

Figure 5. Optimal open-loop profiles for Problem (6) obtained with a direct simultaneous method (DSM) and a parsimonious input model (PIM).

A parsimonious input parameterization can be introduced to reduce the computational load of the online control problem. In this model, the temperature $T(t)$ is parameterized using the switching times $t_{1}$ and $t_{2}$ and a linear profile between the lower and upper bounds between these switching times. On the other hand, the feedrate input $u(t)$ is set to keep the pressure at its upper limit.

It can be seen from Eq. (6) that the pressure constraint has relative degree 1. In other words, the input becomes explicit after the first time derivative of this constraint. Hence, the value of $u(t)$ that keeps the total pressure active can be computed from $\dot{p}_{\text {total }}(t)=\dot{p}_{1}(t)+\dot{p}_{2}(t)=0$, 
which gives $u(t)=V_{l i q}\left(j_{1}{ }^{G L}(t)+j_{2}{ }^{G L}(t)\right)$. As a result, the vector of decision variables for the reformulated problem is simply $\pi=\left(t_{1}, t_{2}\right)^{T}$. Accordingly, the parsimonious input model reads:

$$
\mathcal{U}(\pi)=\left\{\begin{array}{lr}
T(\pi)=\left\{\begin{array}{lr}
368.15 & \text { if } 0 \leq t<t_{1} \\
368.15+\frac{20}{t_{2}-t_{1}}\left(t-t_{1}\right) & \text { if } t_{1} \leq t<t_{2} \\
388.15 & \text { if } t_{2} \leq t<t_{f}
\end{array}\right. \\
u(t)=V_{\text {liq }}\left(j_{1}{ }^{G L}(t)+j_{2}{ }^{G L}(t)\right) & \forall t \in\left[0, t_{f}\right]
\end{array}\right.
$$

The optimal profiles obtained via the parsimonious input model are also shown in Fig. 5, with the optimal cost being 0.589 [mol/L]. Although the input profiles are somewhat different, very similar cost values are achieved $[26,66]$. On the other hand, note that the number of decision variables is reduced from 500 to 2 through the use of the parsimonious input parameterization, which will result in a significant reduction in online computational effort.

\subsubsection{Closed-Loop Simulations with sh-NMPC}

The performance and robustness of the two sh-NMPC schemes are compared under the parametric variations given in Table 2 . The controller sampling time is $30 \mathrm{~s}$. It is assumed that the concentrations of each component can be measured via online spectroscopy every $30 \mathrm{~s}$, with a $5 \mathrm{~s}$ measurement delay. The total pressure in the gas phase is assumed to be measured every second with no delay. Moreover, all measurements are corrupted with Gaussian white noise. A linear observer is used to estimate the concentrations of all species in the liquid phase at each sampling instant [59].

In order to reject the effects of parametric uncertainty on the pressure path constraint, a hierarchical control structure is recommended. In this scheme, the total pressure in the gas phase $p_{\text {total }}$ computed via optimization is sent as a setpoint to a low-level PID controller that tracks the 
pressure by adjusting the feedrate of syngas $u(t)$. This way, the fast perturbations affecting the path constraint are rejected via the low-level controller, while the slow perturbations on the cost are reduced through the upper level sh-NMPC. Note that implicit control of this path constraint via the system equations is also possible, but additional simulations are necessary to determine the values of the back-off terms in the presence of uncertainty and measurement noise [59]. The suggested hierarchical structure is illustrated in Fig. 6, with the thermostat and the state estimator hidden inside the reactor.

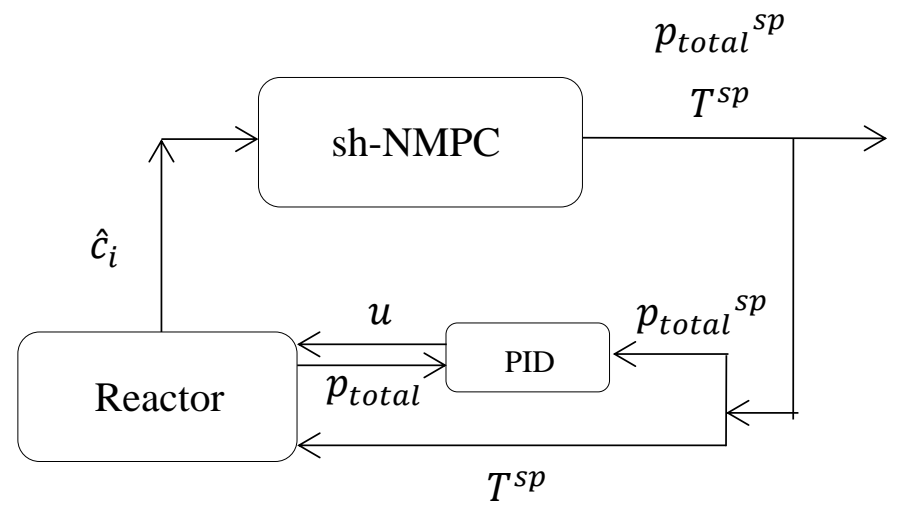

Figure 6. Hierarchical sh-NMPC structure for the semi-batch hydroformylation reactor.

Remark 3. The $\Delta T_{\max }$ constraint can be enforced directly with standard sh-NMPC. With parsimonious sh-NMPC, this constraint can be included in the optimization via a constraint on the two switching times, namely, $t_{2}-t_{1}>15 \mathrm{~min}$.

To be able to compare the performance of the two sh-NMPC methods, closed-loop simulations were performed using the same parametric variations throughout the operation. Also, to have a fair comparison in terms of CPU times, the inputs of standard sh-NMPC were parameterized with 50 piecewise-constant elements. The results are reported in Fig. 7. 

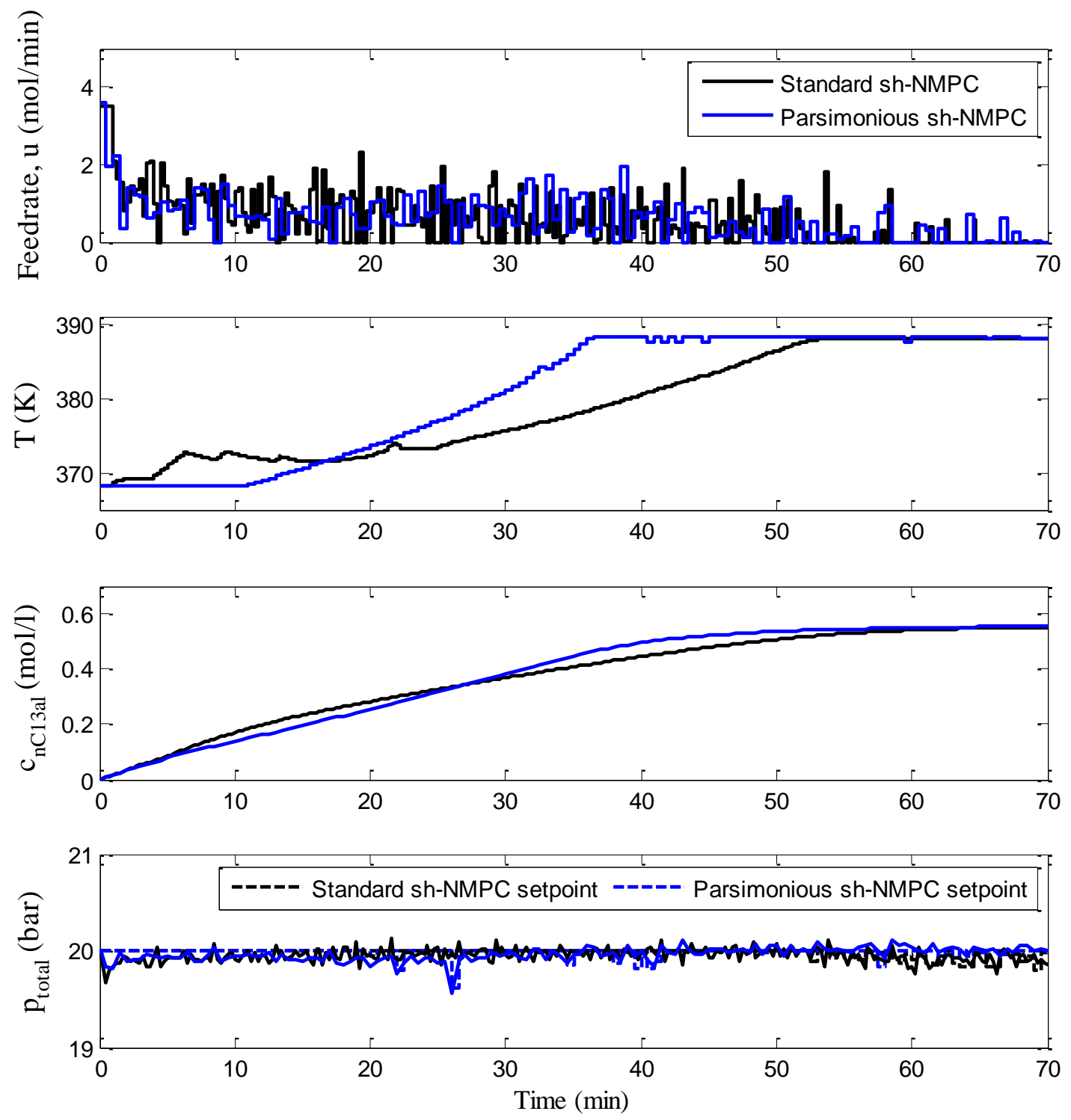

Figure 7. Optimal closed-loop profiles for Problem (6) obtained with standard and parsimonious sh-NMPC.

As shown in Fig. 7, both control schemes give very similar final concentrations of tridecanal (nC13al), even though the input profiles are somewhat different. Furthermore, one sees that the temperature profiles exhibit the same 3-arc shape as the nominal solution. The resulting optimal 
costs are $0.5527[\mathrm{~mol} / \mathrm{L}]$ for standard sh-NMPC and $0.5525[\mathrm{~mol} / \mathrm{L}]$ for parsimonious sh-NMPC. Parsimonious sh-NMPC is computationally far superior to standard sh-NMPC. The CPU times at each sampling time are reported in Fig. 8. One may expect that the computational time reduction associated with parsimonious sh-NMPC leads to improved closed-loop performance due to increased optimization frequency.

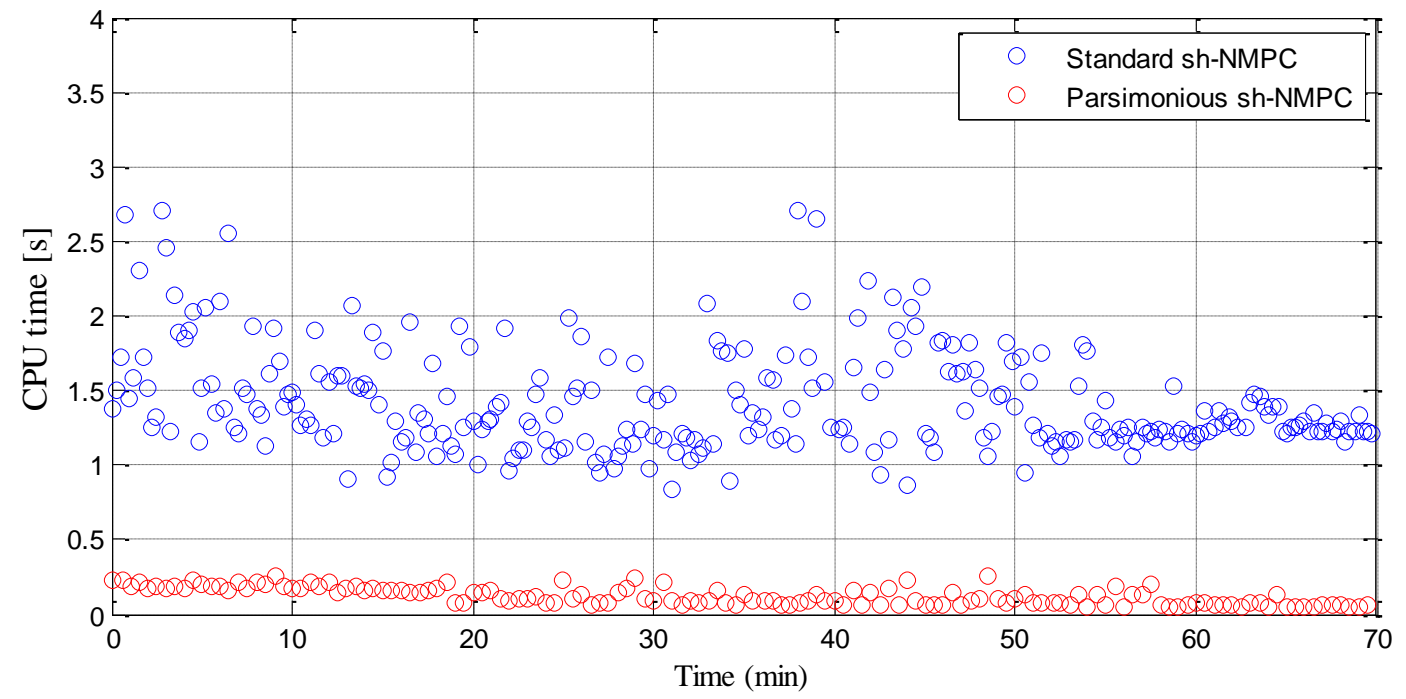

Figure 8. Computational times with standard and parsimonious sh-NMPC.

Finally, parsimonious sh-NMPC is tested for 40 different uncertainty realizations. The optimal profiles are given in Fig. 9. The parsimonious sh-NMPC scheme results in feasible operation, with a mean final concentration of tridecanal of 0.5562 [mol/L]. 

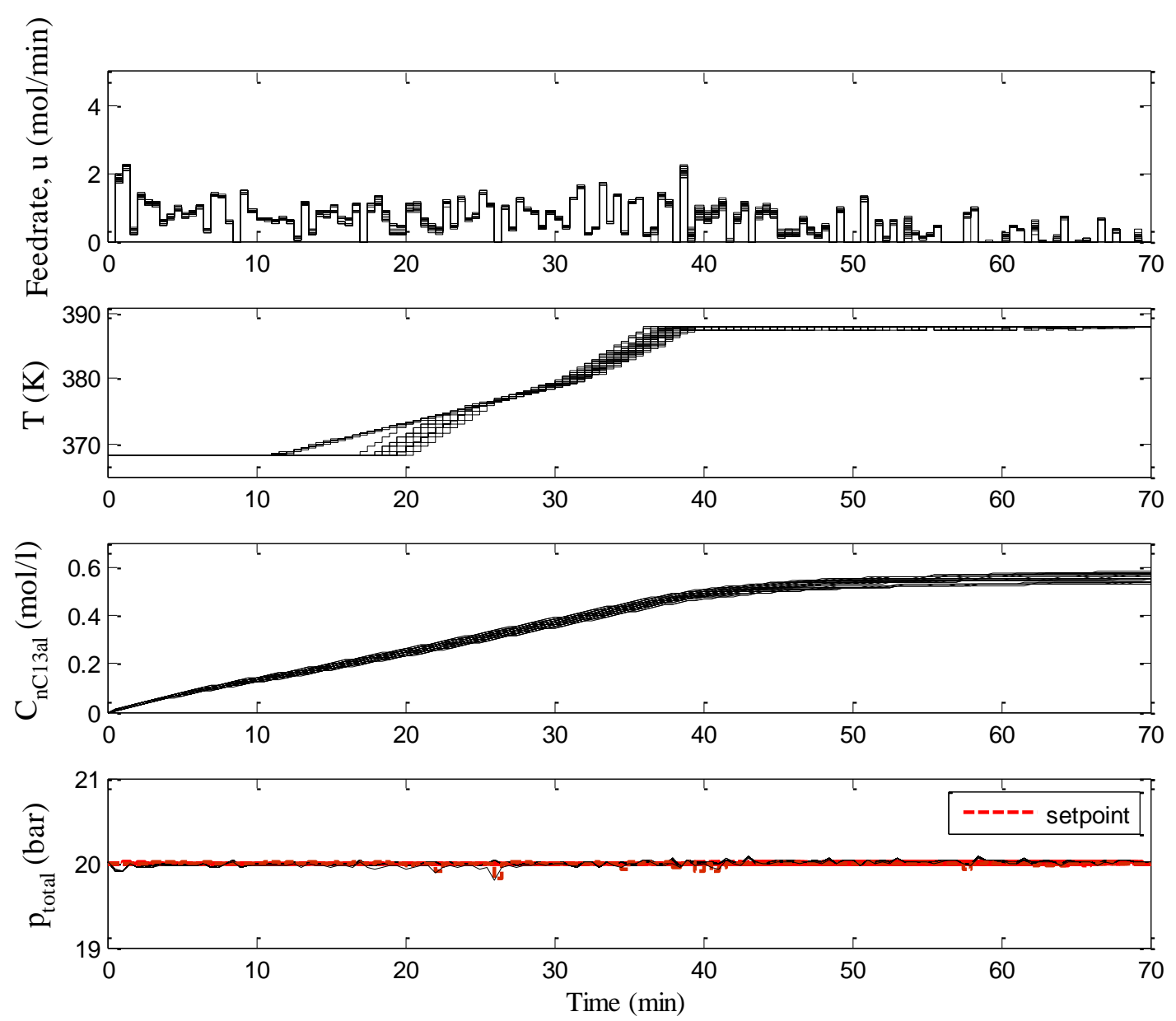

Figure 9. Optimal closed-loop profiles for Problem (6) obtained with parsimonious sh-NMPC for 40 different batches. 


\section{Conclusions}

This study has discussed the use of parsimonious input models together within shrinkinghorizon NMPC for batch processes. This approach reduces the complexity of the optimization problem and therefore also the CPU times. This decrease in online computational effort is important in practice. Faster computation enables higher optimization frequencies, which in turn may lead to better closed-loop performance. Furthermore, faster computations are welcome in real-time embedded systems in which short battery life is a challenge. The resulting scheme, labeled 'parsimonious sh-NMPC', has been applied to two case studies simulated in the presence of uncertainty.

Parsimonious sh-NMPC has been compared to standard sh-NMPC in terms of both nominal performance and robustness. Although the input profiles computed with parsimonious sh-NMPC and standard sh-NMPC differ to some extent, both NMPC schemes exhibit the same solution structures and arc types. It turns out that the performance of parsimonious sh-NMPC is very close to that of standard sh-NMPC in terms of optimal cost. Furthermore, significant reduction in realtime computational effort has been observed in the case studies. Hence, the computational superiority of the parsimonious sh-NMPC scheme makes it very suited to optimize batch processes. 


\section{Acknowledgements}

This work was conducted in part in cooperation with the Collaborative Research Centre "Integrated Chemical Processes in Liquid Multiphase Systems". The financial support from the Deutsche Forschungsgemeinschaft (DFG) under the grant SFB/TRR 63 is gratefully acknowledged. EA acknowledges fruitful discussions with Jens Bremer and Andreas Himmel from Max Planck Institute Magdeburg, and with Diogo Rodrigues from EPFL.

\section{References}

[1] S.J. Qin, T.A. Badgwell, A survey of industrial model predictive control technology, Control Engineering Practice, 11 (2003) 733-764.

[2] M. Morari, J.H. Lee, Model predictive control: Past, present and future, Computers \& Chemical Engineering, 23 (1999) 667-682.

[3] V. Adetola, M. Guay, Integration of real-time optimization and model predictive control, Journal of Process Control, 20 (2010) 125-133.

[4] G. De Souza, D. Odloak, A.C. Zanin, Real time optimization (RTO) with model predictive control (MPC), Computers \& Chemical Engineering, 34 (2010) 1999-2006.

[5] R. Scattolini, Architectures for distributed and hierarchical model predictive control-A review, Journal of Process Control, 19 (2009) 723-731.

[6] S. Engell, Feedback control for optimal process operation, Journal of Process Control, 17 (2007) 203-219.

[7] M. Ellis, P.D. Christofides, Economic model predictive control with time-varying objective function for nonlinear process systems, AIChE Journal, 60 (2014) 507-519.

[8] L. Würth, J.B. Rawlings, W. Marquardt, Economic dynamic real-time optimization and nonlinear model-predictive control on infinite horizons, IFAC Proceedings Volumes, 42 (2009) 219-224.

[9] M. Ellis, H. Durand, P.D. Christofides, A tutorial review of economic model predictive control methods, Journal of Process Control, 24 (2014) 1156-1178.

[10] F. Martinsen, L.T. Biegler, B.A. Foss, A new optimization algorithm with application to nonlinear MPC, Journal of Process Control, 14 (2004) 853-865.

[11] A. Marchetti, M. Amrhein, B. Chachuat, D. Bonvin, Scale-up of batch processes via decentralized control, IFAC Proceedings Volumes, 39 (2006) 221-226.

[12] Z.K. Nagy, B. Mahn, R. Franke, F. Allgöwer, Real-time implementation of nonlinear model predictive control of batch processes in an industrial framework, in: Assessment and Future Directions of Nonlinear Model Predictive Control, Springer, 2007, pp. 465-472.

[13] D. Bonvin, Optimal operation of batch reactors-A personal view, Journal of Process Control, 8 (1998) 355-368. 
[14] D. Bonvin, B. Srinivasan, D. Hunkeler, Control and optimization of batch processes: Improvement of process operation in the production of specialty chemicals, IEEE Control Systems Magazine, 26 (2006) 34-45.

[15] B. Srinivasan, S. Palanki, D. Bonvin, Dynamic optimization of batch processes: I. Characterization of the nominal solution, Computers \& Chemical Engineering, 27 (2003) 1-26.

[16] J. Kadam, M. Schlegel, B. Srinivasan, D. Bonvin, W. Marquardt, Dynamic optimization in the presence of uncertainty: From off-line nominal solution to measurement-based implementation, Journal of Process Control, 17 (2007) 389-398.

[17] E. Visser, B. Srinivasan, S. Palanki, D. Bonvin, A feedback-based implementation scheme for batch process optimization, Journal of Process Control, 10 (2000) 399-410.

[18] Z.K. Nagy, R.D. Braatz, Open-loop and closed-loop robust optimal control of batch processes using distributional and worst-case analysis, Journal of Process Control, 14 (2004) 411422.

[19] J.W. Eaton, J.B. Rawlings, Feedback control of chemical processes using on-line optimization techniques, Computers \& Chemical Engineering, 14 (1990) 469-479.

[20] D. Ruppen, C. Benthack, D. Bonvin, Optimization of batch reactor operation under parametric uncertainty — Computational aspects, Journal of Process Control, 5 (1995) 235-240.

[21] C. Welz, B. Srinivasan, D. Bonvin, Measurement-based optimization of batch processes: Meeting terminal constraints on-line via trajectory following, Journal of Process Control, 18 (2008) 375-382.

[22] M. Diehl, H.G. Bock, J.P. Schlöder, R. Findeisen, Z. Nagy, F. Allgöwer, Real-time optimization and nonlinear model predictive control of processes governed by differentialalgebraic equations, Journal of Process Control, 12 (2002) 577-585.

[23] A. Helbig, O. Abel, W. Marquardt, Model predictive control for online optimization of semibatch reactors, in: American Control Conference, 1998. Proceedings of the 1998, IEEE, 1998, pp. 1695-1699.

[24] D. Ruppen, D. Bonvin, D. Rippin, Implementation of adaptive optimal operation for a semibatch reaction system, Computers \& Chemical engineering, 22 (1998) 185-199.

[25] O. Abel, W. Marquardt, Scenario-integrated on-line optimisation of batch reactors, Journal of Process Control, 13 (2003) 703-715.

[26] E. Aydin, D. Bonvin, K. Sundmacher, Dynamic optimization of constrained semi-batch processes using Pontryagin's minimum principle-An effective quasi-Newton approach, Computers \& Chemical Engineering, 99 (2017) 135-144.

[27] J. Bosley, T. Edgar, Application of nonlinear model predictive control to optimal batch distillation, IFAC Proceedings Volumes, 25 (1992) 303-308.

[28] H. Seki, M. Ogawa, S. Ooyama, K. Akamatsu, M. Ohshima, W. Yang, Industrial application of a nonlinear model predictive control to polymerization reactors, Control Engineering Practice, 9 (2001) 819-828.

[29] J. Valappil, C. Georgakis, Nonlinear model predictive control of end-use properties in batch reactors, AIChE Journal, 48 (2002) 2006-2021.

[30] A. Mesbah, A.E. Huesman, H.J. Kramer, Z.K. Nagy, P.M. Van den Hof, Real-time control of a semi-industrial fed-batch evaporative crystallizer using different direct optimization strategies, AIChE Journal, 57 (2011) 1557-1569.

[31] S. Lucia, T. Finkler, S. Engell, Multi-stage nonlinear model predictive control applied to a semi-batch polymerization reactor under uncertainty, Journal of Process Control, 23 (2013) 13061319. 
[32] H. Jang, J.H. Lee, L.T. Biegler, A robust NMPC scheme for semi-batch polymerization reactors, IFAC-PapersOnLine, 49 (2016) 37-42.

[33] A. Zubov, O. Naeem, S.O. Hauger, A. Bouaswaig, F. Gjertsen, P. Singstad, K.D. Hungenberg, J. Kosek, Bringing the on-line control and optimization of semibatch emulsion copolymerization to the pilot plant, Macromolecular Reaction Engineering, (2017).

[34] M. Cannon, Efficient nonlinear model predictive control algorithms, Annual Reviews in Control, 28 (2004) 229-237.

[35] M. Cannon, W. Liao, B. Kouvaritakis, Efficient MPC optimization using Pontryagin's minimum principle, International Journal of Robust and Nonlinear Control, 18 (2008) 831-844.

[36] R. Findeisen, F. Allgöwer, Computational delay in nonlinear model predictive control, IFAC Proceedings Volumes, 37 (2004) 427-432.

[37] I.J. Wolf, W. Marquardt, Fast NMPC schemes for regulatory and economic NMPC-A review, Journal of Process Control, 44 (2016) 162-183.

[38] V.M. Zavala, L.T. Biegler, The advanced-step NMPC controller: Optimality, stability and robustness, Automatica, 45 (2009) 86-93.

[39] J. Jäschke, X. Yang, L.T. Biegler, Fast economic model predictive control based on NLPsensitivities, Journal of Process Control, 24 (2014) 1260-1272.

[40] E. Suwartadi, V. Kungurtsev, J. Jäschke, Sensitivity-based economic NMPC with a pathfollowing approach, Processes, 5 (2017) 8.

[41] A. Wächter, L.T. Biegler, On the implementation of an interior-point filter line-search algorithm for large-scale nonlinear programming, Mathematical Programming, 106 (2006) 25-57.

[42] R. Huang, V.M. Zavala, L.T. Biegler, Advanced step nonlinear model predictive control for air separation units, Journal of Process Control, 19 (2009) 678-685.

[43] V.M. Zavala, C.D. Laird, L.T. Biegler, A fast moving horizon estimation algorithm based on nonlinear programming sensitivity, Journal of Process Control, 18 (2008) 876-884.

[44] L. Biegler, X. Yang, G. Fischer, Advances in sensitivity-based nonlinear model predictive control and dynamic real-time optimization, Journal of Process Control, 30 (2015) 104-116.

[45] C. Welz, B. Srinivasan, A. Marchetti, D. Bonvin, N. Ricker, Validation of a solution model for the optimization of a binary batch distillation column, in: American Control Conference, 2005, pp. 3127-3132.

[46] C. Welz, B. Srinivasan, A. Marchetti, D. Bonvin, N. Ricker, Evaluation of input parameterization for batch process optimization, AIChE Journal, 52 (2006) 3155-3163.

[47] M. Schlegel, K. Stockmann, T. Binder, W. Marquardt, Dynamic optimization using adaptive control vector parameterization, Computers \& Chemical Engineering, 29 (2005) 1731-1751.

[48] Dynamic optimization of constrained semi-batch processes using Pontryagin's minimum principle and parsimonious parameterization. Computer Aided Chemical Engineering (Vol. 40, pp. 2041-2046): Elsevier.

[49] L.T. Biegler, An overview of simultaneous strategies for dynamic optimization, Chemical Engineering and Processing: Process Intensification, 46 (2007) 1043-1053.

[50] L.T. Biegler, Nonlinear Programming: Concepts, Algorithms, and Applications to Chemical Processes, SIAM, 2010.

[51] E. Aydin, D. Bonvin, K. Sundmacher, NMPC using Pontryagin's minimum principleApplication to a two-phase semi-batch hydroformylation reactor under uncertainty, Computers \& Chemical Engineering, 108 (2018) 47-56.

[52] B. Srinivasan, D. Bonvin, Real-time optimization of batch processes by tracking the necessary conditions of optimality, Industrial \& Engineering Chemistry Research, 46 (2007) 492504. 
[53] M. Schlegel, W. Marquardt, Detection and exploitation of the control switching structure in the solution to dynamic optimization problems, Journal of Process Control, 16 (2006) 275-290.

[54] J. Andersson, M. Diehl, Dynamic optimization with CasADi, 51st IEEE Conference on Decision and Control, 2012, pp. 681-686.

[55] R. Schneider, C. Georgakis, How to not make the extended Kalman filter fail, Industrial \& Engineering Chemistry Research, 52 (2013) 3354-3362.

[56] C.V. Rao, J.B. Rawlings, Constrained process monitoring: Moving-horizon approach, AIChE Journal, 48 (2002) 97-109.

[57] B. Hentschel, G. Kiedorf, M. Gerlach, C. Hamel, A. Seidel-Morgenstern, H. Freund, K. Sundmacher, Model-based identification and experimental validation of the optimal reaction route for the hydroformylation of 1-dodecene, Industrial \& Engineering Chemistry Research, 54 (2015) 1755-1765.

[58] N.M. Kaiser, R.J. Flassig, K. Sundmacher, Probabilistic reactor design in the framework of elementary process functions, Computers \& Chemical Engineering, 94 (2016) 45-59.

[59 O. Abel, A. Helbig, W. Marquardt, H. Zwick, T. Daszkowski, Productivity optimization of an industrial semi-batch polymerization reactor under safety constraints, Journal of Process Control, 10 (2000) 351-362.

[60] J.C. Binette, B. Srinivasan, D. Bonvin, On the various local solutions to a two-input dynamic optimization problem, Computers \& Chemical Engineering, 95 (2016) 71-74. 